\title{
Experimenting with the Physicality of Digital Materiality
}

\author{
Chrissy Pretious-Cooney \\ Designer \\ 3 Bermondsey Square, \\ London, SE1 3UN, UK \\ chrissypc@hotmail.co.uk
}

\section{INTRODUCTION}

I wanted to understand the idea of digital materiality and how software cannot be seen, as it has no predetermined visual form. Digital materiality is electrical signals and code, it has no tangible matter, therefore we cannot physically interact with it - therefore it is immaterial (Grombacher 2017). I wanted to render visible the invisible and to understand digital memory/storage as a physical form. It began with the exploration of a USB flashdrive, physically and digitally deconstructing it, to understand the idea of storage and treating memory as an object.

\section{WHAT DOES DIGITAL STORAGE LOOK LIKE?}

I've created a piece of code that creates visual responses through algorithms (from digital files) into tangible matter. I have visualised digital materiality. The digital file is converted into binary via terminal; then the binary data is converted to images in Processing, in which 6 bits are grouped into RGB pixel colour values. The binary data is portrayed into an image file.
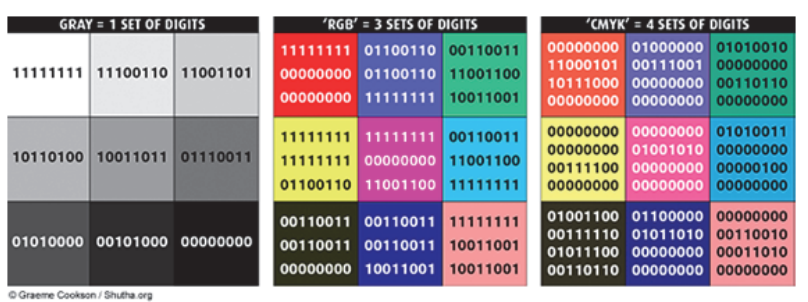

Figure 1: RGB image has three sets of numbers per pixel.

\subsection{The piece}

This particular piece is $15.2 \mathrm{MB}$ of an $81.2 \mathrm{MB}$ song that was performed by my grandma almost 50 years ago. The file is sentimentally valuable and in ways, a memory shared by my family. The piece allows for the 'new aesthetic', a term coined by James Bridle, or a way for us to understand how digital devises see. The pixelated aspect of the piece is not only a representation of a memory or a song but it reflects how machines see and understand the real world (Bridle 2011). The piece is a reflection of a collaboration with technology. My Grandma sings in the real world 50 years ago, it is captured into a recording and then over the years variously digitalised until it is again released into the real world, yet in a different form - merging untouchable virtual data with a tactile object.

I'm looking to expand the boundaries of my project; how much digital memory can I reflect into tangible matter? What form do files take, and what is the significance of the file (song) into physical space? Can we decode the image back into the original binary code? Can I listen to my grandma's song through the tangible piece created?

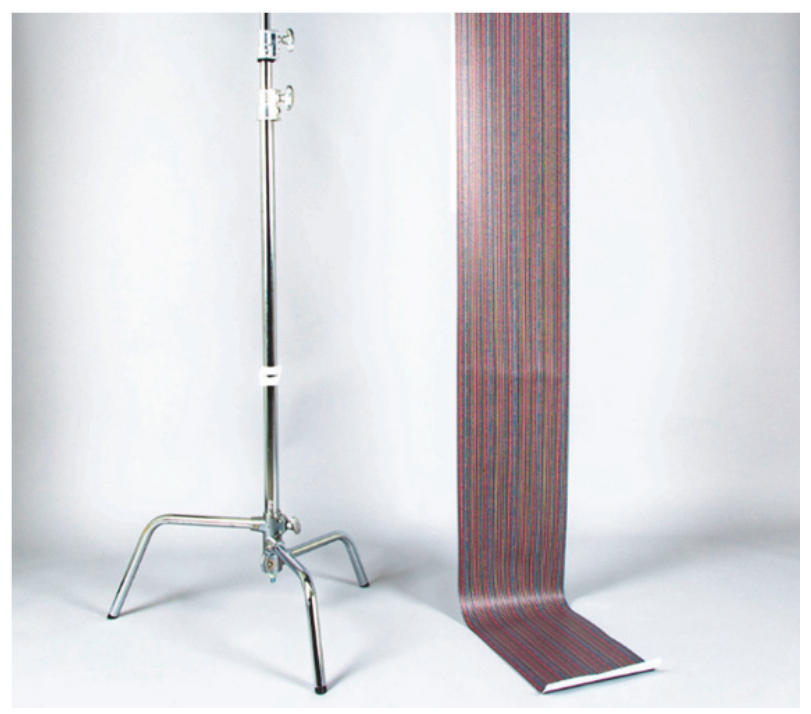

Figure 2: Grandmas Song $(29.7 \mathrm{~cm} \times 500 \mathrm{~cm})$. 
In order for a machine to process sound, it needs to be converted into binary. It works by the sound being captured on a microphone and then converted into a digital signal.

Over the years there have been many debates between which is better, print or digital. There is supposedly such contrast due to explicit differences that people believe there can't be a blur between them, this is digital dualism: the idea that they are two entirely separate things. As we know we have physical things decay and digital non-permanence. Do we care for things more when we know they can be destroyed, we have this idea that digital files are meant to last forever. What happens when we turn bits into atoms, is there a material distinction? Perhaps they are equal, yet different.

If we think of energy, it can neither be created nor destroyed, it just transforms from one form to another. Perhaps it's all just the same no matter what form.

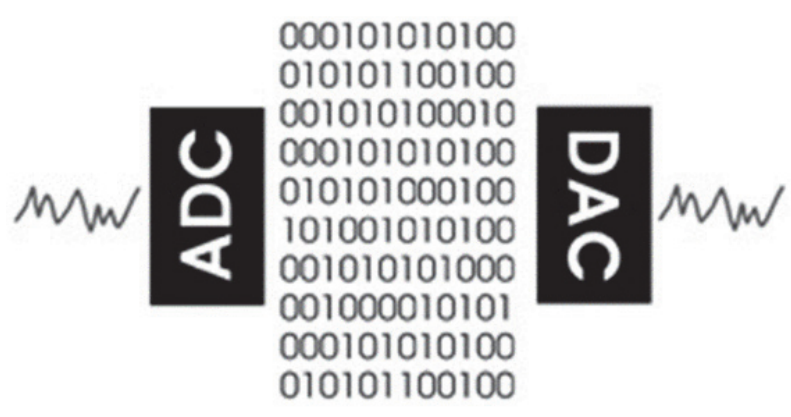

Figure 3: A pictorial description of the recording and playback of sounds through an ADC/DAC.
In analogue to digital conversions, sound waves are copied and displayed as numerical values (binary). In digital to analogue conversions however the binary is put through a converter like a speaker as a vibration so then we can hear it.

My piece of design disrupts this and displays it as an image as oppose to through a speaker to hear. I want it to act as another step between this process, so that we can still eventually hear the song through the speaker. Songs are converted from the analogue to the digital world using a device called an analogy to digital converter (ADC) and the digital to analogy converter (DAC), to convert binary back to sound (Burk et al. 2002).

\section{REFERENCES}

Bridle, J. (2011) James Bridle / The New Aesthetic. https://jamesbridle.com/works/the-new-aesthetic (retrieved 10 March 2019).

Burk, P., Polansky, L., Repetto, D., Roberts, M. and Rockmore, D. (2002) Music and Computers: A Theoretical and Historical Approach. 1st ed. [ebook] Emeryville: Key College Publishing, pp.2.12.8 .

http://sites.music.columbia.edu/cmc/MusicAndCom puters/frontmatter/preface to archival version.php (retrieved 10 March 2019).

Grombacher, P. (2017) What is digital materiality? Rethinking Matter.

http://www.rethinkingmatter.com/blog/2017/3/27/wh at-is-digital-materiality (retrieved 10 March 2019).

Lowe, S. (2015) What does software look like? http://object-mechanics.com/what-does-softwarelook-like/ (retrieved 10 March 2019).

Shutha (2019) Image Pixels. http://shutha.org/node/789 (retrieved 10 March 2019). 